\title{
Pancoast Tumor: A Rare presentation of Hepatocellular Carcinoma
}

\author{
Enam Murshed Khan and Arpita Sutradhar*
}

Dept. of Pathology, Apollo Gleneagles Hospital, Kolkata, India

\begin{abstract}
Lung is the first most common site for metastasis of hepatocellular carcinoma. Pancoast's tumor is usually caused by adenocarcinoma of lung. Hepatocellular carcinoma is very notorious in remaining asymptomatic for prolonged time. But metastatic Hepatocellular carcinoma presenting as apical lobe lung mass eroding the ribs (Pancoast's tumor) is unusual.

We, describe here a 66year old male who presented with left shoulder pain which was subsequently diagnosed to be due to metastatic tumor of upper lobe lung from liver primary.

To our knowledge Pancoast's syndrome due to metastatic hepatocellular carcinoma is rare and only two cases have been reported previously to the best of our knowledge. This case report will allow
\end{abstract}

Keywords: Pancoast Tumor, Hepatocellular Carcinoma, Lung

\section{Introduction}

Pancoast tumors account for less than $5 \%$ of all bronchogenic carcinomas. These tumors are located in the apex of the lung and involve through tissue contiguity the chest wall and/ or the structures of thoracic inlet leading to the symptoms of the "Pancoast Tobias syndrome"(1). Hepatocellular carcinoma metastatic sites include lungs $(55 \%)$, lymph nodes $(53 \%)$, bone( $28 \%)$, adrenal gland(11\%), peritoneum and /or omentum (11\%) and brain(2\%) (2).

Although lung is the commonest site for extrahepatic metastasis of HCC, it presenting as apical lobe mass eroding the ribs is unique. We discuss a 66 year old male having this unique presentation which was subsequently investigated and the diagnosis confirmed.

\section{Case Report}

A 66 year old male presented to the chest clinic with a complain of prolonged left shoulder pain .Considering the age and complain a thorough cardiac check up was done. The 2D Echocardiography studies showed mild left ventricular hypertrophy with good systolic function, Ejection fraction of $59 \%$ and no pulmonary hypertension. Laboratory evaluation revealed AST44U/L,ALT-52U/L,Alk. phosphatase 107U/L.Bilirubin and Total protein were within normal range. Hepatitis $\mathrm{C}, \mathrm{A}, \mathrm{E}$ serology tests were negative but $\mathrm{HbsAg}$ done by ELISA was reactive(4698.16).X-ray chest PA view revealed an ill-defined opacity in the left apex and upper zone with eroded second rib(?Pancoast tumor).Following which PET-CT was done which gave the impression of a heterogeneously enhancing FDG avid solid mass in apicoposterior segment of left lung upper lobe, eroding the left $1^{\text {st }}$ to $3^{\text {rd }}$ ribs (posteriorly) and left transverse processes of D1-D2 vertebra. The mass measured $77 \times 65 \times 64 \mathrm{~mm}$. Extra thoracic extension was seen with infiltration of left upper paraspinal muscle. Live rhad a FDG avid hypodense space occupying lesion measuring $18 \times 12 \mathrm{~mm}$ in segment 5.several FDG avid lytic-sclerotic lesions with enhancing soft tissue components were seen in left ischial tuberosity, right acetabulum, right femur,L5,L4,L2,L1,D9,D8,D7 and C6 vertebrae. PET-CT concluded it to be a active primary malignant disease of left lung upper lobe apico-posterior segment mass with chest wall and vertebral infiltration, along with metastasis to liver and multiple bones. Biopsy was advised for confirmation.

CT guided biopsy offered three linear cores of tissue for histopathological study. Microscopically, there were cores of fibrocollagenous tissue infiltrated by sheets and trabeculae of round to polygonal cells with hyperchromatic pleomorphic nuclei and moderate cytoplasm. Sinusoidal network seen separating and tumor cells. The histomorphology was so that we did immunohistochemistry for HepPar1 which subsequently came out to be strongly positive. Further, immunohistochenmistry markers were done to exclude the possibility of hepatoid Adenocarcinoma of lung i.e TTF1,NapsinA,CK7 and CK5/6 which were all negative. It was also negative for CK20 and CK19.Hence a histopathological diagnosis of Metastatic HCC was made.

Subsequently, a diagnosis of Hepatocellular carcinoma metastasizing to apico-posterior segment of lung and 
thereby presenting as Pancoast tumor was made. Since the disease was at a very advanced stage, he was offered palliative treatment.

\section{Discussion}

Pancoast's tumor is commonly caused by bronchogenic carcinoma. Other causes include other primary thoracic neoplasm, metastatic, including carcinoma of larynx, cervix, urinary bladder and thyroid gland, hematological conditions, infectious diseases, neurogenic thoracic outlet syndromes and pulmonary amyloid nodule(3).But for hepatocellular carcinoma, thoracic involvement presenting as Pancoast tumor is unusual.

The lung is the most common site for extra-hepatic spread, and its involvement is associated with a poor prognosis(4). During necropsy ,52\% of the patients with primary liver carcinoma had evidences of pulmonary metastasis(5). HCC metastasize to lung via hematogenous dissemination through the pulmonary capillary network(6).This results in non calcified soft tissue nodules with lower lobe predominance, Unlike in our case as an atypical apicoposterior lung lobe involvement.
The most useful histopathological diagnostic criteria of HCC are the similarity of tumor cells to liver cells, the prominence of nuclei, the trabecular pattern of growth and sinusoidal stroma(7).In our patient the liver biopsy revealed a typical architecture of $\mathrm{HCC}$, with broad trabecular pattern, sinusoidal vascular channels and a diffuse positivity for HepPar 1 immunostaining.

HCC developes in the setting of chronic liver disease. In the Western world, cirrhosis is present in $85-90 \%$ of $\mathrm{HCC}$ cases, mostly associated with Hepatitis $\mathrm{C}$ virus and alcoholism. The global distribution is linked to prevalence of Hepatitis B virus(8).Many patients are diagnosed late in the disease, owing to the lack of unique symptoms and the livers large functional reserve(9).The closest differential, Hepatoid adenocarcinoma of lung which resembles HCC both histologically and immunohistochemically cannot be ignored. But with such high titres of Hbs Ag and multiple vertebral and bone metastasis the possibility of metastatic $\mathrm{HCC}$ is considered first.

Guidelines from the American Association for the study of Liver diseases recommend screening with ultrasound

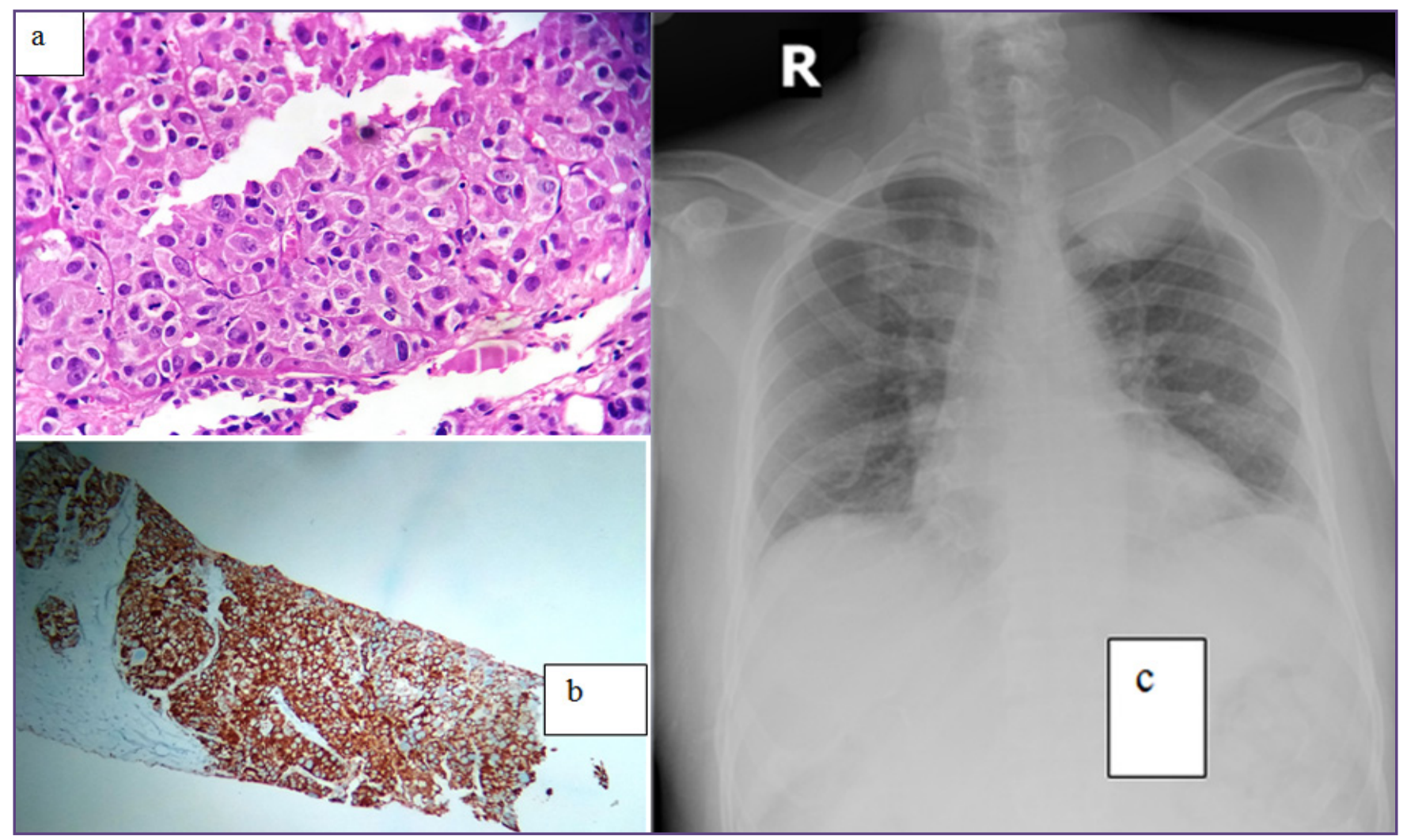

Fig. a): 40X;H\&E.Microscopically tumor is composed of sheets \& trabeculae of polygonal cells with hyperchromatic pleomorphic nuclei and eosinophillic cytoplasm.b) 10X; Immunohistochemically the tumor cells are strongly \& diffusely positive for HepPar1.c) X-Ray chest PA view shows an ill-defined opacity in the left apex and upper zone with eroded second rib(?Pancoast tumor). 
in patients with known Hepatitis B or cirrhosis due to other pathologies(9).Hepatic lesions larger than $1 \mathrm{~cm}$ should be further evaluated with multidetector computed Tomography(CT) or Magnetic Resonance Imaging(MRI) (10).In our case with a high titre of $\mathrm{HbsAg}$, prophylactic imaging studies at regular intervals could have detected $\mathrm{HCC}$ at an earlier stage.

\section{Conclusion}

Metastatic HCC should be included in the differential diagnoses of Pancoast tumor especially in patients with abnormal serology for viral markers or cirrhosis. Metastatic hepatic HCC carries a dismal prognosis and are treated with palliative therapy.

\section{References}

1. Christophoros N, Foroulis, Paul Z et al. Superior sulcus(Pancoast)tumors: current evidence on diagnosis and radical treatment. J Thoracic dis 2013;5(S4):S342-S358.

2. Katyal S, Oliver JH, Peterson MS, et al. Extrahepatic metastases of hepatocellular carcinoma. Radiology 200;216:698-703.
3. Arcasoy SM, Jett JR. Superior pulmonary sulcus tumors and Pancoast's syndrome. N engl J Med 1997;337:1370-6.

4. Shih KL, Chen YY, Teng TH, Soon MS: Long term survival in a patient with repeated resections for lung metastasis after hepatectomy for ruptured hepatocellular carcinoma: a case report. J Med Case Reports 2008,2:222.

5. Honeybourne D: Lung metastases from a primary hepatocellular carcinoma. Thorax 1980,35:316-317.

6. Davis SD. CT Evaluation for pulmonary metastases in patients with extrathoracic malignancy. Radiology 1991;180:1-12.

7. Chao-Fu C, Wei-Juin S, The-Ying $\mathrm{C}$ et al. Hepatocellular Carcinoma with Pancoast's Syndrome as an Initial Symptom: a Case Report. Jpn J Clin Oncol 2001;31(3)119-121.

8. Bosch FX, Ribes J, Cleries R, et al. Epidemiology of hepatocellular carcinoma. Clin Liver Dis2005;9:191-211.

9. Bruix J, Sherman M. Management of hepatocellular carcinoma: an update. Hepatology 2011;53:1020-2.

10. Annalisa K. Becker, David K Tso, Alison C Harris et al. Extrahepatic Metastases of Hepatocellular Carcinoma: A Spectrum of Imaging Findings. Canadian association of Radiologists Journal65(2014)60-66.

*Corresponding author:

Dr Arpita Sutradhar, 58 Canal circular Road, Apollo Gleneagles Hospital, Kolkata-700054 INDIA

Phone: +918420701100

Email: s.arpita3@gmail.com

Financial or other Competing Interests: None. 